Recepción: 20 / 04 / 2017

Aceptación: 20 / 05 / 2017

Publicación: 15 / 06 / 2017

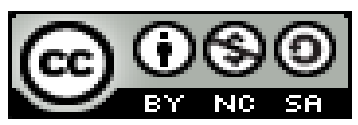

Ciencias Administrativas

Artículo de Investigación

\title{
Gestión administrativa y financiera en el cumplimiento presupuestario de las instituciones públicas
}
Administrative and financial management in the budgetary compliance of public institutions

\section{Gestão administrativa e financeira no desempenho financeiro das instituições públicas}

\author{
Diana C. Escobar-Mayorga ${ }^{\text {I }}$ \\ descobar@utb.edu.ec \\ Julio E. Mora-Aristega II \\ jmora@utb.edu.ec \\ Magdalena R. Huilcapi-Masacón ${ }^{\text {III }}$ \\ mhuilcapi@utb.edu.ec
}

Correspondencia:descobar@utb.edu.ec

I.

Economista, Magister en Administración de Empresas, Docente Universidad Técnica de Babahoyo, Los Ríos, Ecuador.

II.

Licenciado en Ciencias de la Educación Mención Educación Básica, Contador Público Auditor, Magister en Contabilidad y Auditoría, Magister en Docencia y Currículo, Magister en Administración de Empresas, Magister en Gestión de Recursos Humanos, Docente Universidad Técnica De Babahoyo, Los Ríos, Ecuador.

Ingeniera Comercial, Magister en Docencia y Currículo, Magister en Administración de Empresas, Diploma Superior en Diseño Curricular por Competencias, Diploma Superior en Investigación de la Educación a Distancia, Docente Universidad Técnica de Babahoyo, Los Ríos, Ecuador. 


\section{Resumen}

La presente investigación evaluó la gestión administrativa-financiera en el cumplimiento presupuestario de las instituciones públicas del cantón Babahoyo. Se analizaron diferentes opiniones de distintos autores los mismos que ayudaron ampliar el marco teórico de la investigación.

Se utilizó el método de análisis y síntesis mediante encuestas y entrevistas realizada a 52 personas, las mismas que se representan por jefes departamentales y personal administrativo de las áreas de financiero, presupuesto y tesorería, esto permitió evaluar cómo se ha manejado la gestión administrativa-financiera de las instituciones.

Entre los principales resultados se destaca que el cumplimiento de efectividad de las instituciones públicas ha ido disminuyendo en base a la asignación presupuestaria entregada por el gobierno, el instrumento que ayudó a comprobar la hipótesis de la investigación fue el análisis cuantitativo de la prueba Chi-cuadrado, ya que el valor no sobrepasa el nivel del valor crítico.

Palabras clave: Gestión administrativa; gestión financiera; presupuesto. 


\section{Abstract}

The present investigation evaluated the administrative-financial management in the budgetary compliance of the public institutions of the canton Babahoyo. Different opinions of different authors were analyzed, which helped broaden the theoretical framework of the research.

The method of analysis and synthesis was used through interviews and interviews with 52 people, who were represented by department heads and administrative staff in the areas of finance, budget and treasury. This allowed us to evaluate how the administrative and financial management was handled Of institutions.

Among the main results it is highlighted that the effectiveness of public institutions has been reduced based on the budget allocation provided by the government, the instrument that helped to verify the research hypothesis was the quantitative analysis of the Chi-square test, Since the value does not exceed the level of the critical value.

Key words: Administrative management; Financial management; budget. 


\section{Resumo}

Esta pesquisa avaliou a gestão administrativa e financeira no desempenho financeiro das instituições públicas Babahoyo cantão. diferentes opiniões de diferentes autores que ajudaram a estender foram analisados o quadro teórico da pesquisa.

o método de análise e síntese foi utilizado por meio de pesquisas e entrevistas realizadas 52 pessoas, o mesmo que para os chefes de departamento e pessoal administrativo nas áreas de finanças, orçamento e tesouro são representados, o que permitiu avaliar como ele lidou com a gestão administrativa e financeira instituições.

Entre os principais resultados surgiram de que a eficácia cumprimento, pelas instituições públicas tem vindo a diminuir com base na dotação orçamental fornecido pelo governo, o instrumento que ajudou a testar a hipótese da pesquisa foi a análise quantitativa do teste do qui-quadrado porque o valor não exceda o nível de valor crítico.

Palavras chave: Gestão administrativa; gestão financeira; orçamento. 


\section{Introducción.}

El presupuesto es el principal instrumento de política pública, en el cual se concretizan los objetivos y estrategias, se identifica a las unidades responsables de su ejecución, y debe contener los principios de competencia, universalidad, unidad, especialidad, equilibrio, flexibilidad y publicidad.

En la aplicación de las políticas estatales, la Gestión Administrativa forma parte de los procesos denominados: "Habilidades de apoyo" de la organización, por lo que en la práctica se encarga de administrar los recursos humanos, materiales, tecnológicos y de servicios administrativos; coordinando además los planes, programas y proyectos necesarios para el desarrollo de todos los procesos institucionales encaminados a lograr eficiencia y eficacia en el uso de los recursos que mantienen las instituciones públicas.

El presente trabajo de investigación está basado en el control de la Gestión AdministrativaFinanciera en el cumplimiento presupuestario de las instituciones públicas del cantón Babahoyo.

Las instituciones han venido manejando un control presupuestario empíricamente y esto ha provocado que partidas presupuestarias que deben estar destinadas para obras institucionales, se vean afectadas, ya que sus recursos han sido destinados a otras actividades.

La Dirección Financiera, constituye la columna vertebral de las instituciones públicas del cantón Babahoyo, en el ámbito de la estabilidad económica y representa al sistema financiero institucional, está constituido por los subsistemas o centros de responsabilidades como: Presupuesto, Contabilidad, Tesorería, Bienes, Bodega y Nómina, Subsistemas que coadyuvan a cumplir una serie de objetivos y metas, diseñados en planes estratégicos y vinculado con los objetivos del Plan Nacional del Buen Vivir establecido por el Gobierno Central del Ecuador. 
Las instituciones públicas, cuentan con recursos financieros, reglamentos y una planificación estratégica de desarrollo institucional, los mismos que no son gestionados ni utilizados con la eficiencia y eficacia que se requiere al momento de distribuir correctamente los recursos, esto se debe a que no existe un control interno de los ingresos y gastos en proporción a las necesidades requeridas por los diferentes departamentos de cooperación para el logro de las acciones de la función estratégica institucional.

Para la formulación y ejecución del presupuesto existen varias técnicas, siendo una de ellas la de presupuestos por programas. Esta se relaciona con el planteamiento estratégico institucional y se orienta hacia la ejecución de programas, proyectos y actividades.

Dentro de las instituciones públicas a nivel de América Latina, en torno al control de gestión administrativa-financiera, se ha caracterizado por la aplicación de controles presupuestales a razón de las exigencias gubernamentales de seguimiento de gastos en educación y a las normas de control financiero asumidas por dichas instituciones en el ámbito público. Estas exigencias comenzaron a prevalecer rotundamente a mediados de los años 80 s y principio de los $90 \mathrm{~s}$ a raíz de la racionalización del gasto y a la crisis económica de la mayoría de los países de América Latina.

El perfeccionamiento de la gestión administrativa-financiera de la educación superior en instituciones públicas es generalizado, esto se debe a la incorporación de criterios empresariales para la administración de sus fondos. Este hecho, producto de las restricciones presupuestarias y las exigencias de rendición de cuentas que demandan los estados a nivel latinoamericano, como parte de los procesos de modernización y reformas.

La información presupuestaria, busca revelar con razonabilidad los hechos financieros, económicos y sociales de las instituciones y organismos del sector público, mediante su adecuada 
utilización, se empleen en forma transparente los recursos públicos en procura del beneficio colectivo.

Este trabajo pretende resaltar la importancia del presupuesto como un elemento de planificación, gestión, control y evaluación, expresado en términos económicos-financieros dentro del marco de un plan estratégico, capaz de ser un instrumento o herramienta que promueve la integración en las diferentes áreas que tenga la Institución Pública, la participación como aporte al conjunto de iniciativas dentro de cada centro de responsabilidad y la responsabilidad expresado en términos de programas establecidos para su cumplimiento dentro de una estructura claramente definidos para este proceso.

Dentro del Estado Ecuatoriano, se tiene como marco legal de referencia a la: Constitución Política de la República, normas y acuerdos del control presupuestario, Ley orgánica de la contraloría, y su reglamento, Ley de presupuestos, los mismos que son entregados por el Gobierno Central, Ministerio de Economía y Finanzas y la Contraloría General del estado.

Bajo estos criterios se considerará el capítulo III de la Ley Orgánica de Administración Financiera y Control, en el cual describe como debe estar conformado los métodos y procedimientos del control interno de las Instituciones Públicas.

De acuerdo al art.2 de la ley de regulación económica y control del gasto público resuelve: Los presupuestos de las universidades y escuelas politécnicas, serán aprobados de conformidad con las normas establecidas en su propia ley.

Según las normas de presupuesto actualizadas al 11/10/2011 nos permite analizar la normativa del sistema de Administración Financiera y Presupuesto de las Instituciones públicas. 
Dentro del marco legal de la investigación se considerará el acuerdo 039 de la Contraloría General del Estado, el mismo que contribuirá con las normas de Control Interno considerando un mejor ambiente de control, evaluación del riesgo, administración financiera presupuestaria y administración financiera de tesorería, los mismos que aportarán para un mejor desarrollo del tema en estudio.

Todas estas leyes, normas y acuerdos nos llevaron a un mejor análisis del estudio el que se refiere a la aplicación de los procedimientos del control interno presupuestario.

La presente investigación busca determinar el funcionamiento de la gestión administrativa y financiera en el cumplimiento presupuestario de las instituciones públicas del cantón Babahoyo.

\section{Materiales y métodos.}

La investigación que se realizó es de tipo descriptiva y de campo. Cada una de ellas sirvió para comprender y resolver alguna situación, necesidad o problema en un contexto determinado.

El grupo estudiado corresponde a los funcionarios públicos del Cantón Babahoyo.

Se tomó en consideración como población a todos los empleados que conforman el departamento financiero y de tesorería de las instituciones públicas, es decir se trabajó con alrededor de 52 personas las cuales aportaron en el proceso de investigación.

Por tratarse de una población finita se trabajó con el 100\% de la población.

La localización del universo está ubicada en el cantón Babahoyo, particularmente sus instituciones públicas. 
Diana C. Escobar-Mayorga; Julio E. Mora-Aristega; Magdalena R. Huilcapi-Masacón

Se planteó la metodología y las técnicas de investigación, posterior a la aplicación de las encuestas se realizaron los respectivos análisis. Las técnicas utilizadas fueron: Las entrevistas y las encuestas.

Estas técnicas fueron aplicadas a las áreas de estudio, en este caso rector, vicerrector, decanos, sub-decanos, departamento de presupuesto, departamento financiero, tesorería y personal administrativo de los departamentos antes mencionados.

Los métodos y técnicas que se utilizaron en la investigación permitieron ampliar nuestra investigación y determinar la hipótesis. El método que se ha utilizado es el de: análisis y síntesis

En el método de análisis, se distinguieron los elementos del fenómeno y se procede a revisar ordenadamente cada uno de ellos por separado, este método ayudó analizar cada uno de los problemas encontrados en el departamento de presupuesto, permitiendo revisar de forma eficiente los materiales proporcionado por esta área de trabajo.

El método de la síntesis: Ha permitido unificar varias teorías, las cuales se utilizaron las más adecuadas para el tema de investigación. La síntesis se produce sobre la base de los resultados previos del análisis.

Se analizó el universo de las encuestas planteando cuadros y gráficas que reflejan los resultados obtenidos, además del respecto análisis. 


\section{Resultados.}

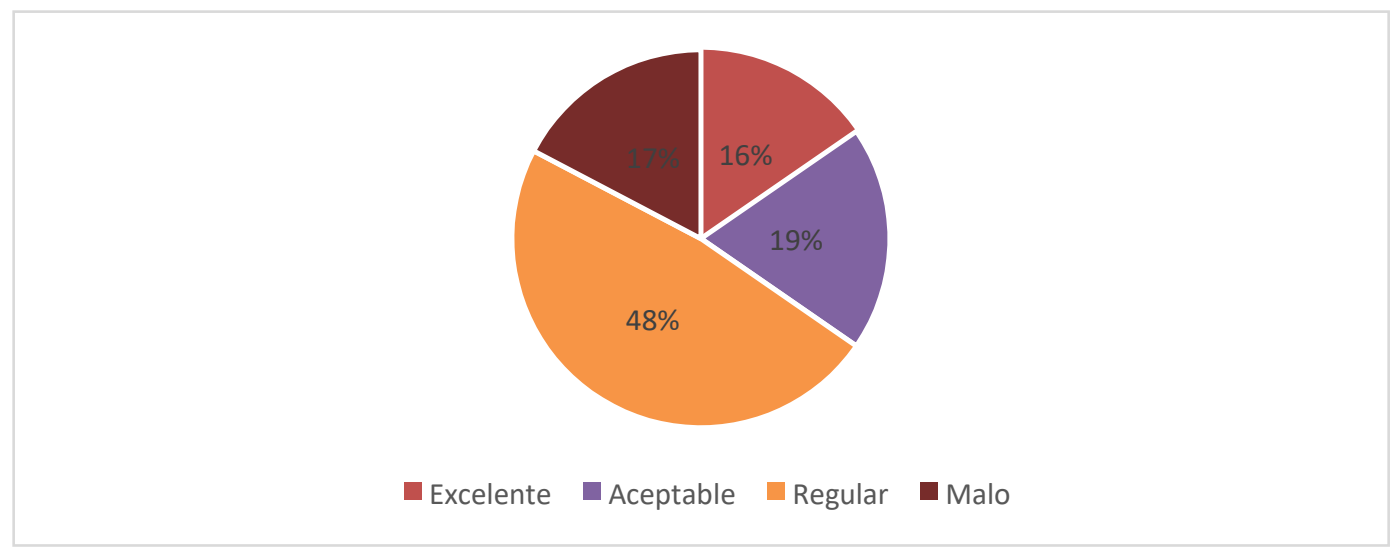

\section{Gráfico $\mathbf{N}^{0} 1$ Control en la gestión administrativa-financiera}

El $48 \%$ considera que el control es regular, mientras que para el $15 \%$ es excelente y un $17 \%$ considera que es malo.

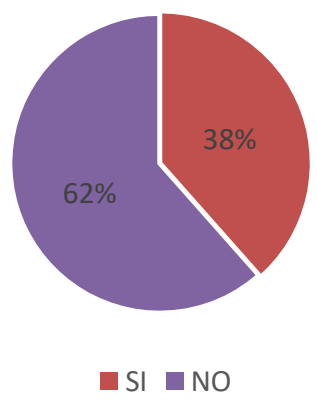

Gráfico No2 Conocimiento de la asignación presupuestaria que entrega el gobierno central a las instituciones públicas.

El 38\% del personal administrativo de las instituciones públicas, conoce la asignación presupuestaria que entrega el gobierno central y el 62\% desconoce está asignación presupuestaria, esto se debe a la falta de comunicación que existe entre los departamentos que deben manejar esta información. 


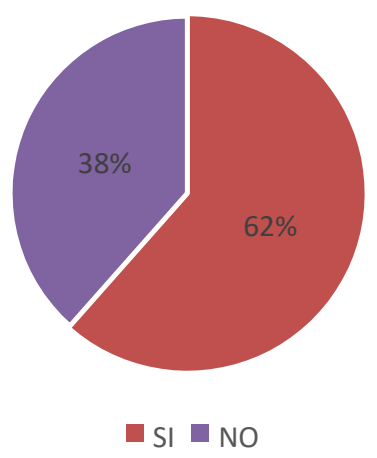

\section{Gráfico No 3: Disponibilidad de herramientas necesarias para el control presupuestario.}

El 38,46\% del personal administrativo indicó que el departamento financiero y de presupuesto no cuentan con las herramientas de control. El 61,54\% indico que cuenta con herramientas pero no son utilizadas eficientemente, lo cual indica que es necesaria la implementaciòn de una unidad que se encargue de llevar el control de la gestión administrativafinanciera para una mejor asignación del recurso económico.

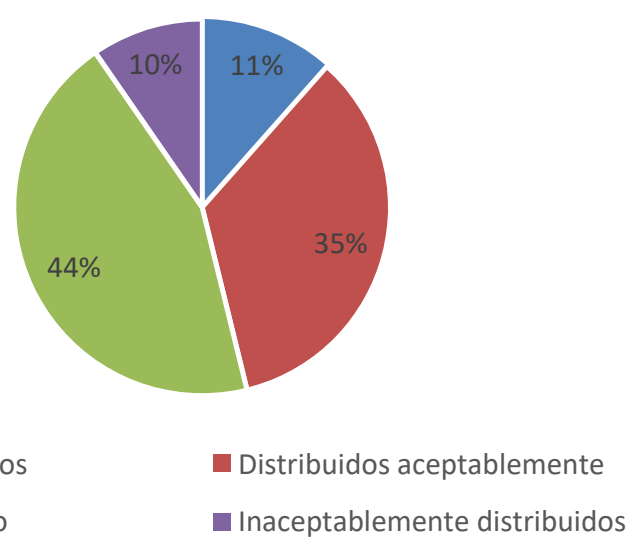

Gráfico No 4: Distribución de recurso financiero. 
El 44\% de los encuestados indicaron que están mal distribuidos los recursos financieros y el $35 \%$ indicaron que esta distribuidos aceptablemente.

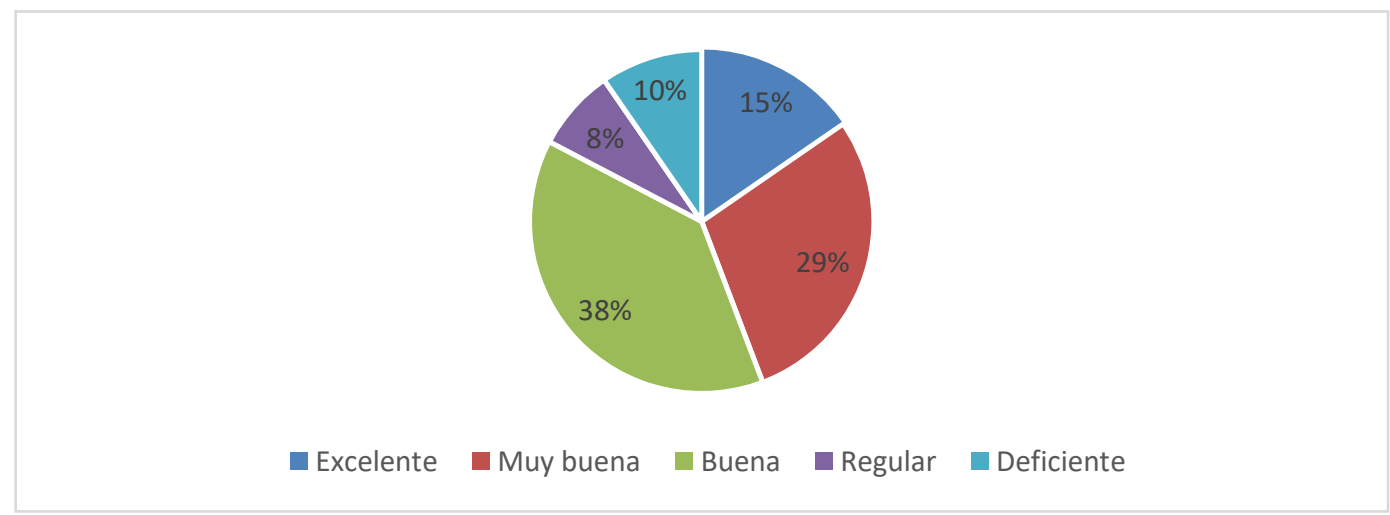

\section{Gráfico $\mathbf{N}^{0} 5$ Calificación de la gestión financiera por autoridades.}

El $38 \%$ considera que la gestión administrativa-financiera por parte de las autoridades fue buena, mientras que el $29 \%$ indican que fue muy buena.

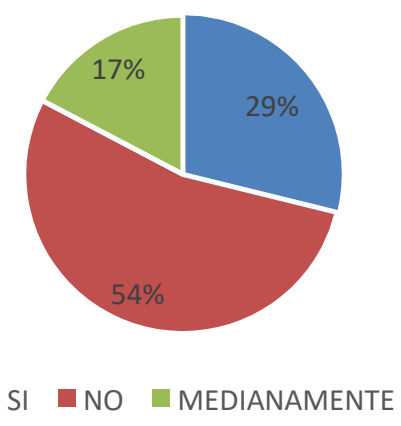

Gráfico No 6 Aplicación de mecanismos de control del departamento financiero. 
Diana C. Escobar-Mayorga; Julio E. Mora-Aristega; Magdalena R. Huilcapi-Masacón

El $53,85 \%$ del personal administrativo indican que los mecanismos de control que fueron utilizados en el departamento financiero no brindaron resultados, mientras que el $28,85 \%$ indicaron que sí.

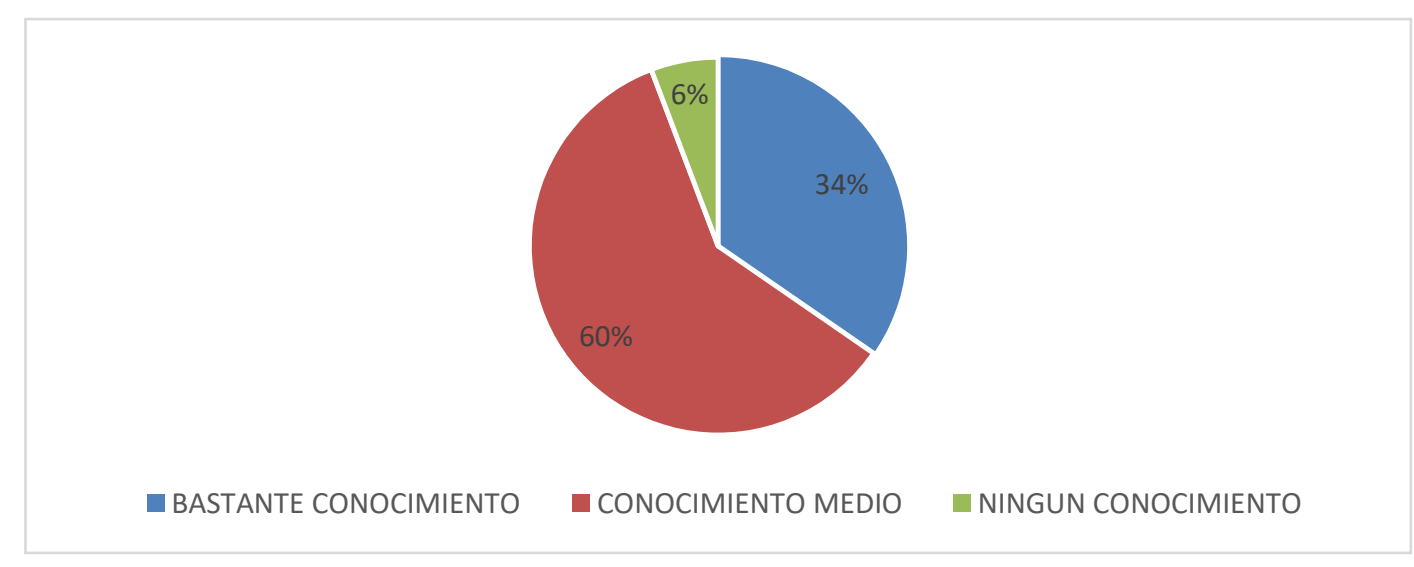

\section{Gráfico $\mathbf{N}^{0} 7$ Conocimiento sobre las normas de control interno.}

El 59,62\% del personal administrativo consideran que los funcionarios financieros tienen un conocimiento medio de las normas de control interno.

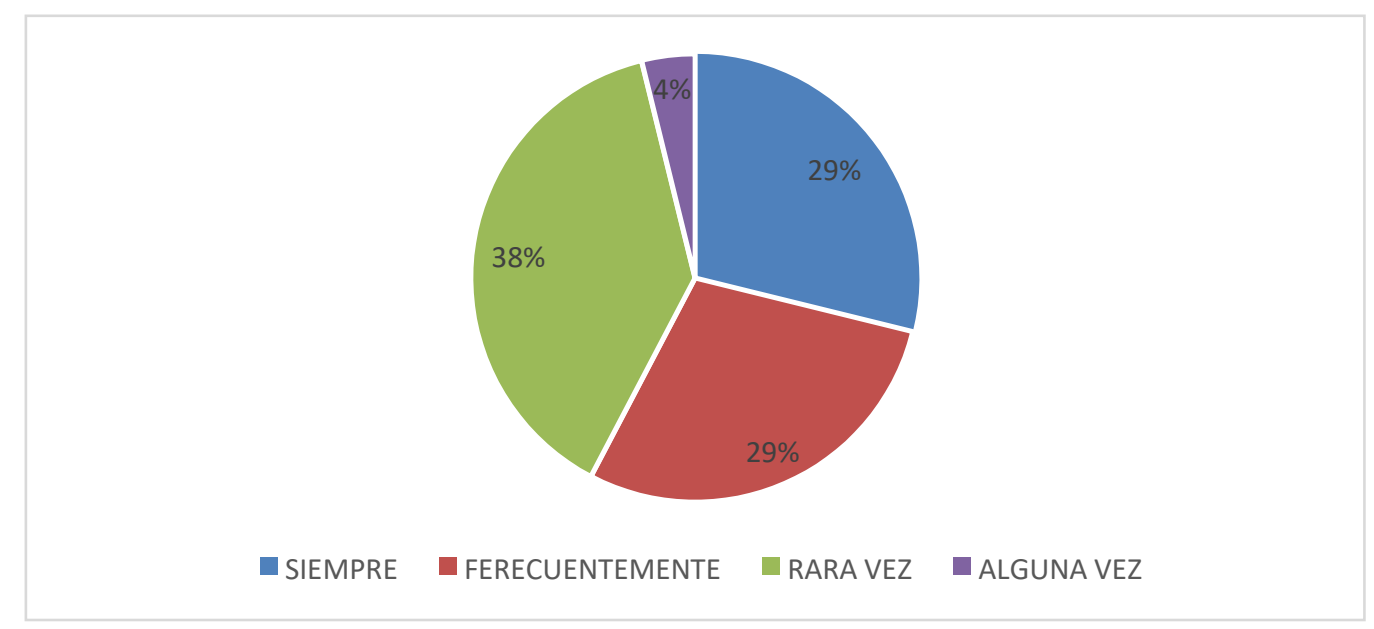

Gráfico No 8 Interrelación entre el departamento de presupuesto, tesorería y el personal administrativo. 
El $38 \%$ de los empleados consideran que rara vez existe la comunicación entre los departamento financiero, presupuesto y tesorería, y el $29 \%$ consideran que frecuentemente existe comunicación entre los departamentos antes mencionados.

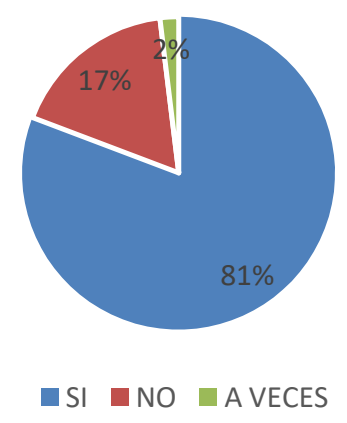

\section{Gráfico $\mathrm{N}^{\circ} 9$ Criterio respecto a la implementación de la unidad de auditoria interna y la gestión administrativa-financiera.}

El 80,77\% del personal administrativo de las instituciones públicas, considera que se debe implementar una unidad de auditoria interna, la misma que aportaría a una mejor gestión administrativa-financiera

\section{Conclusiones.}

- La gestión administrativa-financiera por parte de las autoridades de las instituciones públicas, durante el periodo de investigación se considera regular, lo cual indica que existe una apreciación poco eficiente en el desarrollo de la gestión, para el cumplimiento presupuestario.

- La información entregada a las autoridades sobre la gestión administrativa-financiera, por parte de los jefes departamentales del área financiera ha provocado en algunos casos la toma 
Diana C. Escobar-Mayorga; Julio E. Mora-Aristega; Magdalena R. Huilcapi-Masacón

de decisiones incorrecta y el gasto innecesario del presupuesto, esto se debe a la falta de conocimientos de políticas y normas de control interno institucional.

- La implementación de la unidad de auditoria interna contribuye a mejorar la toma de decisiones y a elevar la efectividad de la ejecución presupuestaria.

\section{Bibliografía.}

Burbano, J. (2011). Presupuesto, un enfoque de direccionamiento estrategico, gestion y control de recursos. MacGrawHill.

Castro, A. O. (2012, Junio). Administracion Financiera. In A. O. Castro, Administracion Financiera. Mck GranHill.

CES. (2010, 10 6). Consejo de Educacion Superior. Retrieved 12 2, 2014, from Consejo de Educacion Superior: http://www.ces.gob.ec/descargas/ley-organica-de-educacion-superior.

CONGRESO NACIONAL DEL ECUADOR. Ley Orgánica de Administración Financiera y Control, LOAFYC.

Duran Herrera, J. (2011). Economia y Direccion Financiera de la Empresa. Piramide.

Española, R. A. (n.d.). Real Academia Española. Real Academia Española.

ESTADO, C. G. (2014). Control Interno Presupuestario. Ecuador: Contraloria General del Estado.

Fayol, H. (2010). Administración. Mexico: Mackgranhill.

Gaitàn, R. E. (2006). Control Interno y Fraudes. Bogota: Ecoe, Ediciones.

Gitman, L. J. (2010). Principios de Administracion Financiera. Addison Wesley.

Heinz, K. H. (2008). Administraciòn,una Perspectiba Global. McGraw-Hill Interamericana.

Idalberto, C. (2008). Introducciòn a la Teoria General de la Administraciòn. McGraw-Hill Interamericana.

Mary, R. S. (2009). Administraciòn. Pearson Educaciòn.

Meyer, J. (2012). Gestion Presupuestaria.

Montalvo, O. G. (2013). Administracion Financiera. MckGranHill.

Muniz, D. (n.d.). El presupuesto.

Muñiz, L. (2011). Presupuesto.

Ormaechea, J. A. (2008). Auditoria y Control Interno. INMAGRAG.

Orozco, S. (2013, Marzo). Scribid. Retrieved Marzo 21, 2014, from Scribid: http://es.scribd.com/doc/50780485/administracion-financiera

Reinaldo, O. D. (2005). Teorias de la Administraciòn. International Thomson Editores, S.A. de C.V. 
Gestión administrativa y financiera en el cumplimiento presupuestario de las instituciones públicas

$(2014$

Marzo

07).

Slideshare.

Retrieved

from

http://www.slideshare.net/mianacru/gestion-administrativa-8839327

Ley de regulación económica y control del gasto público.

Chiavenato, Idalberto (2012). Iniciación a la Organización y Técnica Comercial. Mc Graw Hill/Interamericana de México, México DF.

Rendición Social de Cuentas UTB año 2013.

Durán Herrera, J. J. (2011), “Economía y Dirección Financiera de la Empresa”, Ed. Pirámide, Madrid.

MONTEBELLO, Jorge, Investigación, planeación y evaluación de la Administración Financiera Editorial

IDEA, Buenos Aires, 2011 